\title{
Trauma medular cervical en pacientes con osificación del ligamento longitudinal posterior sin evidencia de fractura. Serie de casos y revisión bibliográfica
}

\author{
Guillermo A. Ricciardi, Ignacio Garfinkel, Gabriel Carrioli, Daniel O. Ricciardi \\ Equipo de Columna Vertebral, Unidad de Ortopedia y Traumatología, Hospital General de Agudos "Dr. Teodoro Álvarez”, \\ Ciudad Autónoma de Buenos Aires, Argentina
}

\begin{abstract}
RESUMEN
Objetivos: Presentar una serie de casos de lesión medular aguda traumática asociada a osificación del ligamento longitudinal posterior cervical sin evidencia tomográfica de trauma óseo y realizar una revisión narrativa de la bibliografía sobre su tratamiento y pronóstico posoperatorio. Materiales y Métodos: Descripción de una serie de pacientes con lesión medular aguda traumática y osificación del ligamento longitudinal posterior cervical, tratados por nuestro equipo, entre enero de 2012 y diciembre de 2019. Se excluyó a pacientes con fracturas o luxaciones vertebrales asociadas, aquellos derivados a otro centro antes del tratamiento y con registros incompletos. Además, se llevó a cabo una revisión narrativa de la bibliografía sobre el tratamiento y el pronóstico posoperatorio de esta asociación en la última década. Con nuestra estrategia de búsqueda, se obtuvieron 10 artículos, a partir de los cuales se desarrolló la revisión narrativa. Resultados: Se conformó una muestra de 5 casos, todos hombres, con una edad promedio de 62.2 años ( $D E \pm 9,36$ ), 4 pacientes fueron operados por vía posterior y uno recibió tratamiento conservador. Conclusiones: La lesión medular aguda traumática asociada a osificación del ligamento longitudinal posterior cervical es infrecuente en países no asiáticos, precedida, en nuestra región, por un reporte de caso aislado. La revisión de la bibliografía actual sugiere al tratamiento quirúrgico oportuno por sobre el tratamiento conservador, pero persisten las controversias al respecto.
\end{abstract}

Palabras clave: Osificación; ligamento longitudinal posterior; lesión medular aguda; trauma.

Nivel de Evidencia: IV

Traumatic Acute Spinal Cord Injury Associated with Ossification of the Cervical Posterior Longitudinal Ligament without Tomographic Evidence of Bone Trauma. Case-Series and Literature Review

\begin{abstract}
Objectives: To present a case series of traumatic acute spinal cord injury associated with ossification of the cervical posterior longitudinal ligament (OPLL) without tomographic evidence of bone trauma and to perform a narrative review of the literature on its treatment and postoperative prognosis. Materials and Methods: We described a case series of patients with traumatic spinal cord injury and association of cervical OLLP, treated by our team during the period January 2012 - December 2019. We excluded patients with an association of vertebral fractures and/or dislocations, and those referred to another center before treatment and with incomplete records. Additionally, a narrative review of the literature on postoperative treatment and prognosis of this association was carried out. Ten articles were obtained with our search strategy for the narrative review. Results: A sample of 5 cases was formed, all males, with an average age of $62.2( \pm 9.36), 4$ cases were surgically treated by a posterior approach and 1 case was conservatively treated. Conclusion: We presented a case series of a rare association in our region, preceded only by an isolated case report. The review of the current literature suggests timely surgical treatment over conservative treatment, but controversies persist in this regard.
\end{abstract}

Keywords: Ossification; posterior longitudinal ligament; spinal cord injury; cervical trauma.

Level of Evidence: IV 


\section{INTRODUCCIÓN}

La lesión medular traumática aún representa un desafío en la atención de pacientes politraumatizados por las controversias asociadas a aspectos diagnóstico-terapéuticos y porque, una vez establecida, configura una lesión "devastadora" de gran morbimortalidad e impacto socioeconómico. ${ }^{1,2}$

Sabemos que una evaluación diagnóstica "a tiempo" condiciona la oportunidad terapéutica, en la cual el hallazgo de fracturas vertebrales inestables o elementos que comprimen el neuroeje alertan al equipo de cirujanos de columna sobre la posibilidad de operar para la liberación de elementos neurológicos, y la reducción y la estabilización de lesiones vertebrales. ${ }^{3}$

Dentro del espectro de las lesiones traumáticas medulares del adulto existen aquellas sin evidencia de fractura ni de luxación vertebral en las radiografías y la tomografía, denominadas SCIWORA, SCIWOCTET o SCIWORET. ${ }^{4-9}$ El término SCIWORA es un acrónimo en inglés de Spinal Cord Injury without Radiological Abnormality, fue acuñado, en 1982, por Pang en pacientes pediátricos evaluados con radiografías y define la lesión traumática de la médula espinal sin evidencia radiográfica o tomográfica de lesión ósea. ${ }^{4,5}$ La tomografía computarizada y la resonancia magnética $(\mathrm{RM})$ permitieron demostrar, en pacientes adultos, la asociación de lesiones no traumáticas vinculables al mecanismo de lesión (calcificación ligamentaria, cambios degenerativos, hernias de disco centrales, estenosis de canal vertebral), sugiriendo que el acrónimo SCIWORA era una denominación "errónea" ${ }^{6,7}$ De hecho, autores con similares consideraciones propusieron términos alternativos, como SCIWOCTET (Spinal Cord Injury Without CT Evidence of Trauma) y SCIWORET (Spinal Cord Injury Without Radiographic Evidence of Trauma). ${ }^{8.9}$

La osificación del ligamento longitudinal posterior (OLLP) es un proceso patológico de deposición de hueso lamelar en la estructura mencionada. ${ }^{10}$ Tiene una alta prevalencia en los países asiáticos, afecta al $2 \%$ de los japoneses, y representa una de las principales causas de canal cervical estrecho. Por tal motivo, las publicaciones sobre lesión medular aguda traumática complicada por OLLP son mayoritariamente de origen asiático. ${ }^{11-21}$

De acuerdo con nuestra búsqueda bibliográfica en revistas indexadas en bases de datos biomédicos nacionales e internacionales, encontramos un único caso reportado de origen hispanoamericano. ${ }^{22}$ En consecuencia, consideramos relevante nuestro aporte como experiencia en el tratamiento de esta grave lesión en un país no asiático.

Nuestro objetivo es presentar una serie de casos de trauma medular cervical asociado a OLLP sin signos tomográficos de fracturas y luxaciones vertebrales.

El objetivo secundario fue efectuar una revisión narrativa de la bibliografía sobre el tratamiento y el pronóstico posoperatorio de este grupo de pacientes.

\section{MATERIALES Y MÉTODOS}

\section{Serie de casos}

Se llevó a cabo un estudio descriptivo de una serie de casos de trauma medular cervical asociado a OLLP, tratados por el mismo equipo quirúrgico, en dos centros: un hospital del sistema público de salud de la Ciudad Autónoma de Buenos Aires y un centro de derivación de patología laboral de la misma región. El período de estudio se prolongó de enero de 2012 a diciembre de 2019.

Como criterio de inclusión se consideró a pacientes con lesión medular aguda traumática objetivable en la RM y evidencia tomográfica de calcificación del ligamento común vertebral posterior en íntima relación con el área medular afectada. Se excluyó a pacientes con evidencia de fracturas o luxaciones vertebrales, a los derivados a otro centro luego de la revisión primaria y aquellos con registros incompletos de historias clínicas.

Se describieron las siguientes variables de estudio: edad, sexo, comorbilidades, antecedente traumático, tiempo preoperatorio ( $>72 \mathrm{~h} \mathrm{o}<72 \mathrm{~h}$ ), estado neurológico inicial según la escala de la American Spinal Injury Association (ASIA), ${ }^{23}$ tipo de OLLP según la clasificación morfológica: (A) continua, (B) segmentaria, (C) combinada y (D) limitada al espacio discal (Figura 1), ${ }^{24}$ extensión de la mielopatía según los niveles cervicales (imagen hiperintensa en la secuencia T2 de RM), tratamiento realizado (quirúrgico o conservador), tipo de cirugía (técnica y vía de abordaje), evolución neurológica posterapéutica (estable, mejoría o deterioro neurológico) y complicaciones (relacionadas con el trauma y la intervención).

En la descripción, se incluyeron los datos individuales, sus respectivas medidas de resumen y dispersión. Se consideraron la media y la desviación estándar para las variables numéricas, y el valor absoluto y el porcentaje para las nominales. 


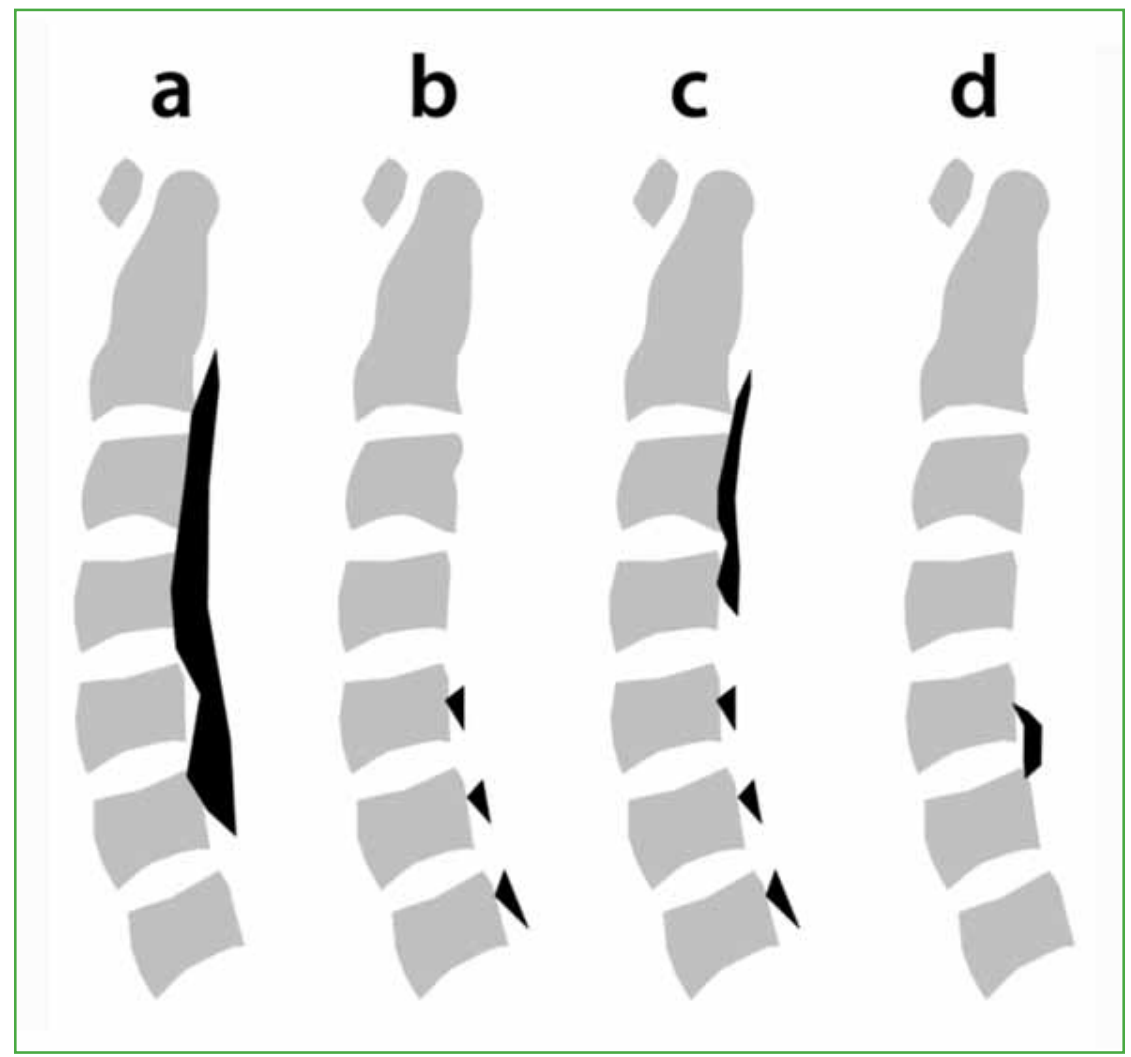

Figura 1. Clasificación morfológica de la osificación del ligamento longitudinal posterior en 4 tipos: tipo A o continua (extendida a lo largo de varias vértebras), tipo B o segmentaria (áreas de osificación segmentadas en el muro posterior del cuerpo vertebral), tipo C o mixta (combinación de continua y segmentada) y tipo D (limitada al espacio discal).

Revisión narrativa de la bibliografía sobre el tratamiento y el pronóstico posoperatorio de la lesión medular aguda complicada por la OLLP

Se llevó a cabo una revisión bibliográfica en las principales bases de datos biomédicas MEDLINE, EMBASE, LILACS, Cochrane Library a través de los buscadores PubMed, portal de la BVS y Google Académico, considerando como período de estudio artículos publicados entre enero de 2010 y septiembre de 2020, mediante las siguientes palabras clave y estrategias de búsqueda:

- Estrategia 1: ((((“cord injury”) AND (“ossification of the posterior longitudinal ligament”)) AND (cervical)) AND (trauma)) AND (treatment)

- Estrategia 2: ((((“cord injury”) AND (“ossification of the posterior longitudinal ligament”)) AND (cervical)) AND (trauma)) AND ("outcomes")

En el buscador PubMed, se recolectaron 22 artículos (estrategia 1: 20; estrategia 2: 10; coincidencias: 8). Se excluyeron reportes de caso, estudios de pacientes con fracturas o luxaciones vertebrales asociadas y opiniones de expertos (13 artículos). Se seleccionaron nueve de todos los artículos obtenidos por este medio. ${ }^{11,18-20,25-29}$ Se obtuvo un artículo adicional a través de Google Académico con las mismas palabras clave. ${ }^{30}$ Finalmente, se incluyeron 10 artículos en la revisión. Se registraron las variables: autor principal, año de publicación, país de origen, diseño del estudio y nivel de evidencia. 


\section{RESULTADOS}

\section{Serie de casos}

Durante el período de estudio, se incluyeron siete pacientes con lesión medular aguda traumática y OLLP. Dos pacientes fueron excluidos: uno por derivación a otro centro luego de la atención inicial y uno por óbito en las primeras $24 \mathrm{~h}$ antes del tratamiento con falta de registro de datos en la historia clínica. Se conformó una muestra de cinco pacientes. Todos eran hombres, con una media de la edad de 62.2 años (desviación estándar 9.36).

Como antecedente traumático, dos (40\%) habían sufrido una caída desde su propia altura; dos (40\%), una colisión motovehicular y uno (20\%), un accidente ecuestre.

Todos tenían un déficit neurológico inicial severo según la escala ASIA: cuatro (80\%) ASIA A y uno (20\%) ASIA C (síndrome medular central).

Como antecedentes de relevancia, tres $(60 \%)$ pacientes tenían comorbilidades (obesidad: 2 casos, hipertensión arterial y diabetes: 2 casos) y uno (20\%), lesiones asociadas al trauma (trauma cerrado de tórax); ninguno tenía antecedentes de mielopatía.

Según la clasificación morfológica de la OLLP, un caso (20\%) era tipo A o continua (caso 1); uno (20\%), tipo B o segmentaria (caso 2); uno (20\%), tipo C o combinada (caso 5); dos (40\%), tipo D o limitada al espacio discal (casos 3 y 4 ) (Figuras 2 y 3 ).

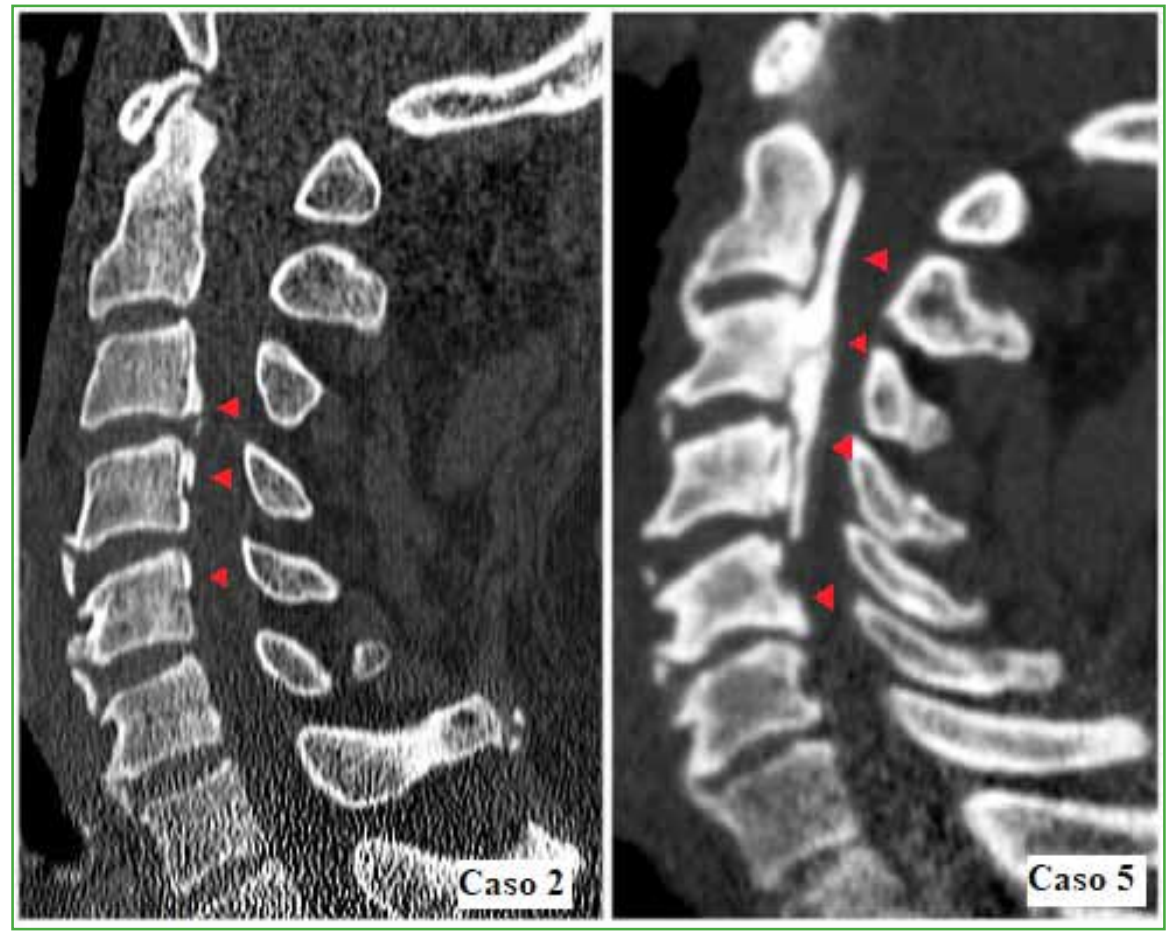

Figura 2. Tomografía computarizada, corte sagital. Se observan imágenes características de osificación del ligamento longitudinal posterior (flechas) segmentaria (Caso 2) y mixta (Caso 5).
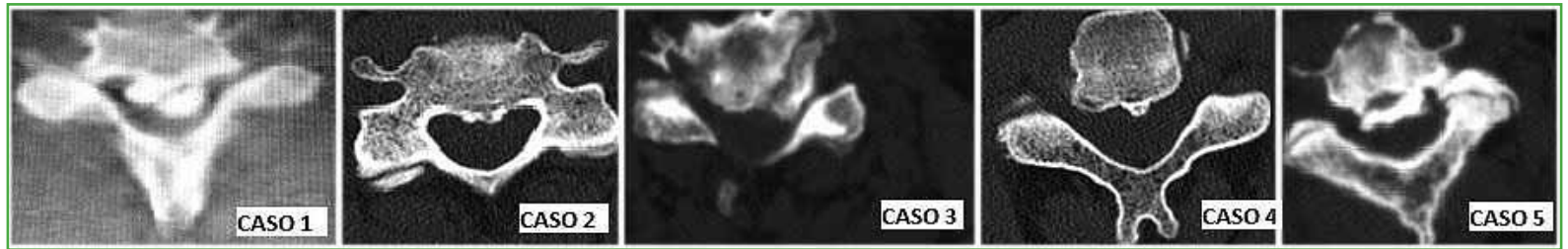

Figura 3. Tomografía computarizada, corte axial. Casos 1-5. Imágenes compatibles con osificación del ligamento longitudinal posterior. 
Todos los pacientes tenían imágenes de topografía medular hiperintensas en la secuencia de T2 de RM de gran extensión, con compromiso de múltiples niveles cervicales (Figura 4).

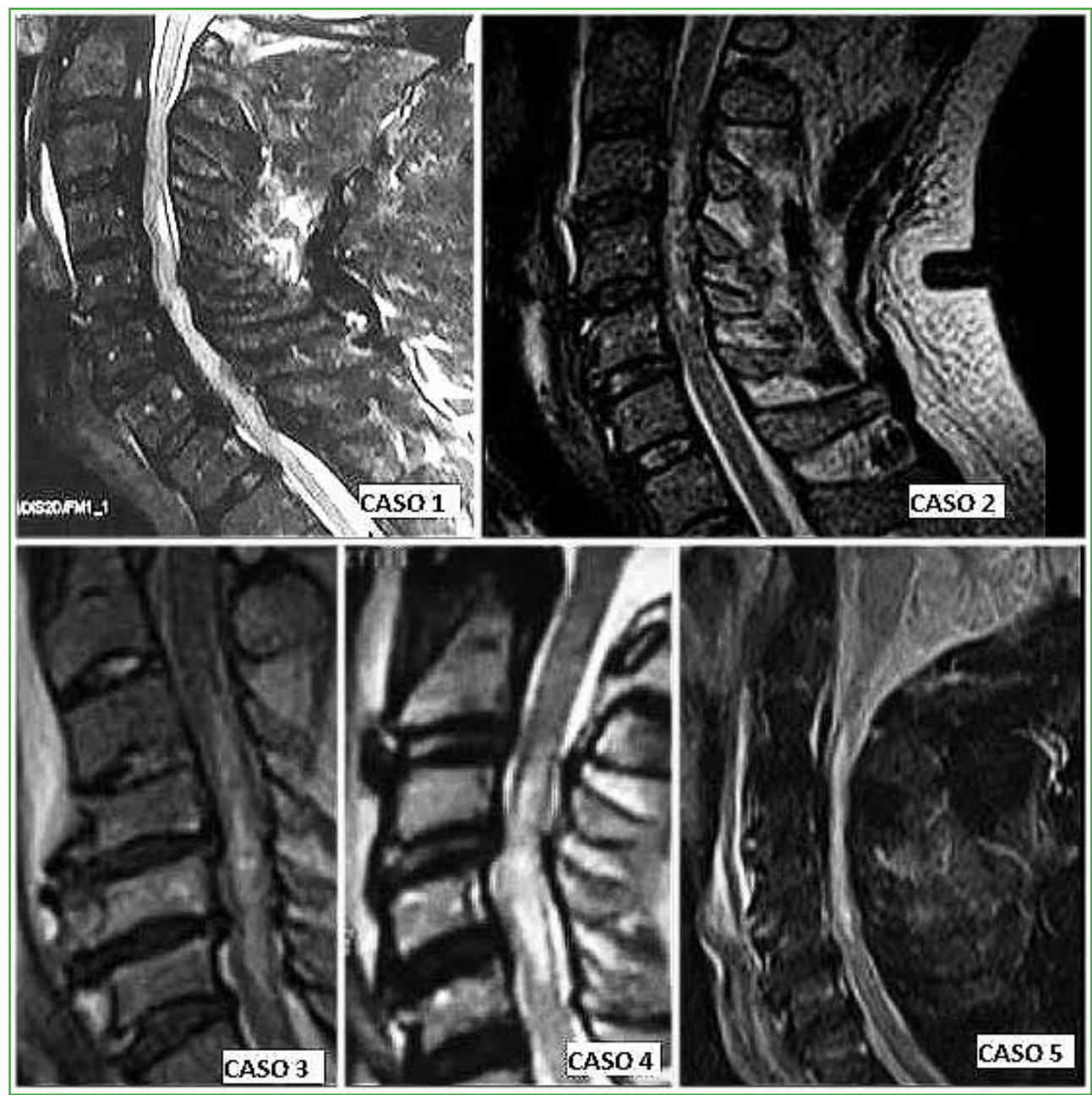

Figura 4. Resonancia magnética inicial. Casos 1-5. Aumento de la señal medular en la secuencias T2 (Caso 2) y STIR (Casos 1, 3, 4 y 5).

Se describen cuatro (80\%) pacientes tratados mediante cirugía y uno (20\%) que recibió tratamiento conservador. Tres fueron operados antes de las $72 \mathrm{~h} \mathrm{y}$, en el paciente restante, fue necesario diferir la cirugía más allá de este plazo por dificultad respiratoria asociada complicada por una infección intrahospitalaria. Todas las cirugías fueron por vía posterior. Las técnicas quirúrgicas implementadas fueron laminoplastia (1 caso), laminectomía y artrodesis ( 2 casos) y laminectomía sin fijación (1 caso). La elección de la técnica quirúrgica se basó en el análisis de los estudios complementarios, la disponibilidad de implantes a la brevedad en la urgencia y la preferencia del cirujano (Figura 5). 


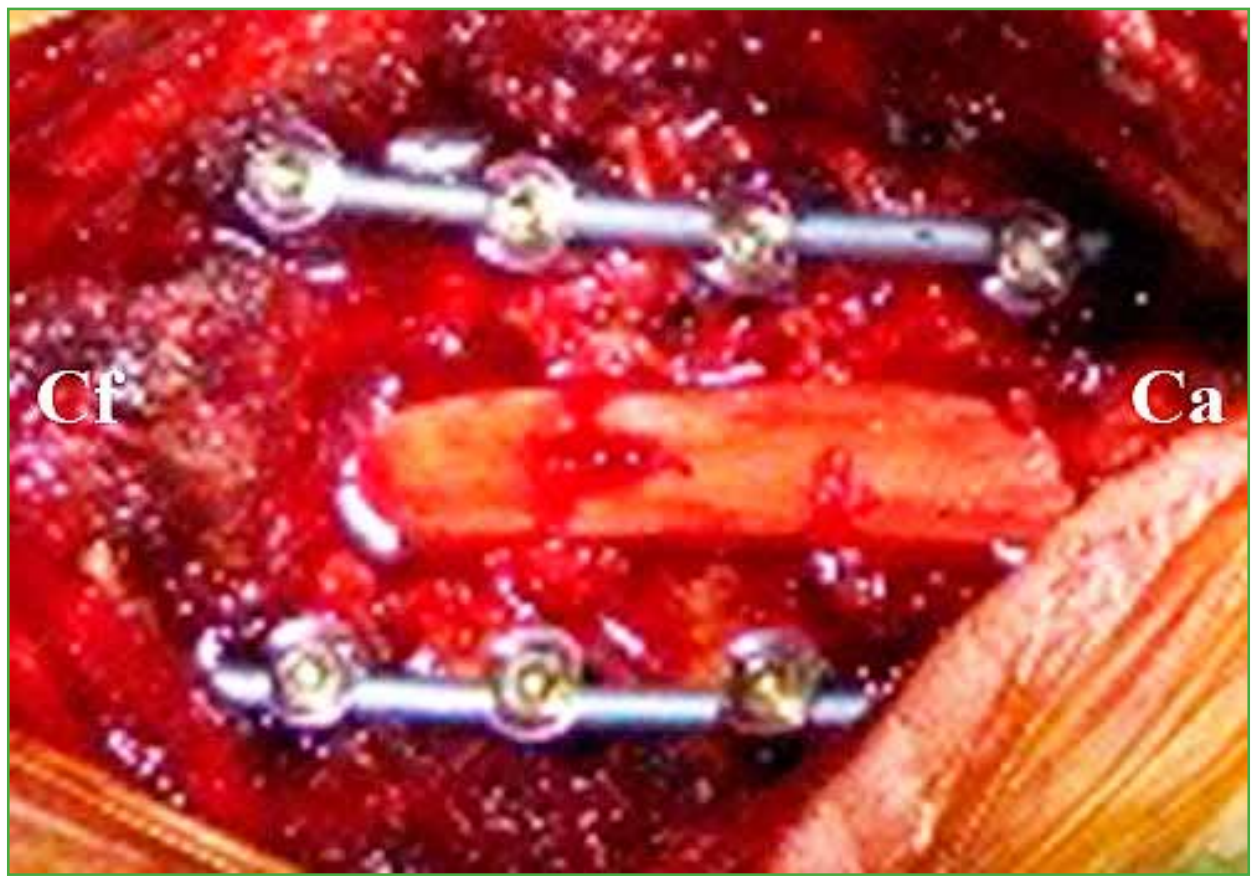

Figura 5. Imagen intraoperatoria. Laminectomía y artrodesis. $\mathrm{Cf}=$ cefálico, $\mathrm{Ca}=$ caudal.

Un paciente recibió tratamiento conservador por propia decisión y la de su familia frente a los riesgos de la cirugía y el pronóstico de la lesión medular (caso 5, ASIA A).

Durante el posoperatorio inmediato, dos pacientes presentaron compromiso hemodinámico significativo (shock neurogénico) que provocó el óbito de uno de ellos a las 48 h de la cirugía y del otro una semana después.

Un paciente falleció a los seis meses a causa de un cuadro gastrointestinal no asociado con el trauma (divertículo perforado y peritonitis fecal). La tasa de mortalidad total fue del 60\%, el $40 \%$ ( 2 casos) murió en el posoperatorio inmediato. Dos pacientes continúan en rehabilitación y seguimiento hasta la actualidad.

El estado neurológico no mejoró en ningún caso, en un tiempo de seguimiento promedio de 3.9 años $( \pm 3.78)$, en tres de los cinco casos.

Todos recibieron un esquema de corticoides por vía intravenosa con metilprednisolona al ingresar, según el protocolo NASCIS II.

En la Tabla 1, se resume la descripción de las variables y, en la Tabla 2, los datos de los pacientes.

\section{Revisión narrativa de la bibliografía}

En la Tabla 3, se detallan los artículos incluidos en la revisión bibliográfica.

\section{Factores de riesgo de lesión medular aguda traumática en pacientes con OLLP}

Wu y cols. estudiaron una cohorte retrospectiva de pacientes con OLLP tratados de forma conservadora frente a un grupo de control sin OLLP en un período de ocho años de seguimiento. La incidencia de lesión medular cervical fue significativamente más elevada en la cohorte de pacientes con OLLP que en el grupo de control, con una incidencia de 4,81 frente a 0,18 pacientes por cada 1000 personas-año. Este estudio no valoró el riesgo de mielopatía asociado a aspectos específicos de la OLLP (tipo, diámetro del canal, edad de los pacientes). ${ }^{29}$

Onishi y cols. estimaron retrospectivamente una mayor asociación entre OLLP y lesión medular aguda en pacientes de edad avanzada, aquellos con patrón de osificación mixta o segmentaria y la presencia adicional de osificación del ligamento común vertebral anterior. ${ }^{20}$ 
Tabla 1. Descripción de la serie

Media de la edad (DE)

$62.2( \pm 9,36)$

$$
\begin{gathered}
\text { Sexo, n (\%) } \\
\text { Masculino } \\
\text { Femenino }
\end{gathered}
$$

$5(100)$

$0(0)$

Antecedente traumático

baja energía, n (\%)

$2(40)$

alta energía, n (\%)

$3(60)$

Lesión neurológica inicial, n (\%)

ASIA A

ASIA B

$4(80)$

ASIA C

$0(0)$

ASIA D

$1(20)$

$0(0)$

Clasificación de la OLLP, n (\%)

Tipo A

$1(20)$

Tipo B

1 (20)

Tipo C

1 (20)

Tipo D

$2(40)$

Extensión de la imagen medular en la resonancia magnética (T2), n (\%) de $\mathrm{C} 4$ a $\mathrm{T} 1$

de $\mathrm{C} 3$ a $\mathrm{C} 5$

1 (20)

de $\mathrm{C} 2$ a $\mathrm{C} 5$

2 (40)

1 (20)

de $\mathrm{C} 3$ a C6

1 (20)

Tratamiento quirúrgico, n (\%)

Sí

$4(80)$

No

1 (20)

Cirugía, n (\%)

Laminoplastia

$1(20)$

Laminectomía

$1(20)$

Laminectomía y artrodesis

$2(60)$

Evolución neurológica, n (\%)

Mejoría

Estable

Deterioro

OLLP $=$ Osificación del ligamento longitudinal posterior.

Tabla 2. Datos de los pacientes

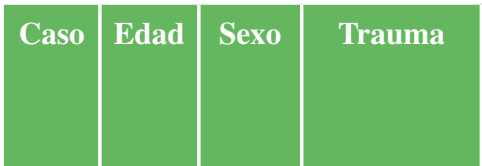

1<smiles>[125IH]</smiles>

$2 \quad 55$

$55 \mathrm{M}$

Caída desde propia altura

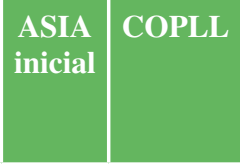

A

A

A B

C3-C5

propia altura

C D

C3-C6

C3-C5

A D

A

C2-C5

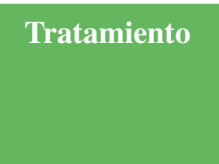

Quirúrgico

Quirúrgico

Quirúrgico

Conservador

Quirúrgico
Laminectomía $\mathrm{y}$ artrodesis

Laminectomía

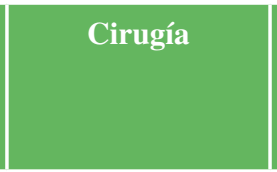

Laminoplastia

Laminectomía y artrodesis
Estable

Estable

Estable

Estable

Evolución

neurológica

Estable 
Tabla 3. Artículos sobre osificación del ligamento longitudinal posterior (OLLP) y lesión medular aguda traumática

\begin{tabular}{|c|c|c|c|c|}
\hline Año & Autor & Origen & Tipo de estudio & $\begin{array}{l}\text { Nivel de } \\
\text { evidencia }\end{array}$ \\
\hline 2011 & Yan y cols. ${ }^{25}$ & China & Serie de casos & IV \\
\hline 2011 & Chikuda y cols. ${ }^{11}$ & Japón & Retrospectivo analítico observacional. Multicéntrico & III \\
\hline 2012 & Onishi y cols. ${ }^{20}$ & Japón & $\begin{array}{l}\text { Retrospectivo, analítico observacional. } \\
\text { Comparación de } 3 \text { grupos: 1) lesión medular aguda y OLLP, } \\
\text { 2) mielopatía y OLLP y 3) control }\end{array}$ & III \\
\hline 2012 & Wu y cols. ${ }^{29}$ & Taiwán & Retrospectivo, analítico observacional de comparación & III \\
\hline 2014 & Choi y cols. ${ }^{28}$ & Corea del Sur & Retrospectivo, analítico observacional, correlación & III \\
\hline 2014 & Gu y cols. ${ }^{18}$ & China & Retrospectivo, analítico observacional, comparación 2 grupos & III \\
\hline 2015 & Kwon y cols. ${ }^{26}$ & Corea del Sur & Retrospectivo, analítico observacional, análisis multivariado & III \\
\hline 2016 & Gu y cols. ${ }^{19}$ & China & Retrospectivo, analítico observacional & III \\
\hline 2020 & Li y Jiang ${ }^{30}$ & China & Retrospectivo, analítico observacional, análisis multivariado & III \\
\hline 2020 & $\begin{array}{l}\text { Hollenberg y } \\
\text { Mesfin }^{27}\end{array}$ & Estados Unidos & Retrospectivo, analítico observacional, comparación & III \\
\hline
\end{tabular}

\section{Tratamiento quirúrgico frente a tratamiento conservador}

El tratamiento de la lesión medular aguda traumática asociado a OLLP continúa siendo controvertido. No hay estudios publicados con un elevado nivel de evidencia que guíen su terapéutica.

En un estudio retrospectivo multicéntrico de 34 centros de Japón, Chikuda y cols. evaluaron a 94 pacientes. La mejoría neurológica fue mayor en pacientes tratados con cirugía; sin embargo, no fue estadísticamente significativa. Destacan que, cuando segmentaron la muestra según la existencia de compromiso de la marcha anterior al trauma (mielopatía previa), en este subgrupo, la mejoría con el tratamiento quirúrgico fue significativa. ${ }^{11}$

En un estudio retrospectivo de 60 pacientes, Gu y cols. obtuvieron una mayor proporción de casos con recuperación neurológica en el seguimiento de pacientes tratados con cirugía frente a los que recibieron tratamiento conservador. ${ }^{18}$

Yang y cols. publicaron una serie de 25 pacientes con mejoría del estado neurológico en 21 de los 25 casos. Veinte pacientes de la serie tenían cuadros neurológicos Frankel C o D. ${ }^{25}$

\section{Factores pronósticos del resultado del tratamiento quirúrgico}

En la última década, se han publicado cinco estudios retrospectivos con el objetivo de estimar los factores pronósticos del tratamiento quirúrgico en pacientes con lesión medular complicada por la OLLP.

Kwon y cols. evaluaron, en forma retrospectiva, los factores asociados con la recuperación neurológica posoperatoria en una serie de 38 pacientes tratados por vía posterior (laminoplastia o laminectomía) en un período de siete años. Mediante un análisis multivariado estimaron que la edad avanzada, un menor puntaje motor de la escala de ASIA al ingresar, la gravedad de la hiperintensidad medular en la secuencia T2 de la RM preoperatoria y un menor espacio disponible para la médula (SAC) se asociaron con peores resultados neurológicos. ${ }^{26}$

Gu y cols., en un estudio de diseño similar con 36 pacientes operados por diferentes vías de abordaje, estimaron la presencia de una zona de hiperintensidad de señal medular en la secuencia T2 de la RM como principal factor asociado a peores resultados en la recuperación neurológica posoperatoria. ${ }^{19}$

En un análisis multivariado retrospectivo de 69 pacientes con lesión medular traumática aguda sin evidencia de fractura o luxación vertebral que incluyó 10 pacientes con OLLP, Li y Jiang no demostraron una relación entre el pronóstico de la lesión medular y la presencia de OLLP. Los factores de asociación significativa con el pronóstico posoperatorio fueron: 1) el puntaje ASIA inicial (los pacientes ASIA C y D al ingreso tuvieron una tasa más alta de recuperación neurológica), 2) la longitud de la lesión medular en la RM ( $\geq 45 \mathrm{~mm}$ se asoció con peores resultados neurológicos), 3) la relación de Pavlov ( $<0,65$ se asoció con pobres resultados) y 4) el tipo de lesión medular en la RM (la coexistencia de sangrado y edema se asoció con peores resultados). ${ }^{30}$ 
Choi y cols. compararon los resultados obtenidos con la cirugía de liberación posterior (laminectomía o laminoplastia) en pacientes con mielopatía cervical de curso crónico y aquellos con antecedentes traumáticos de baja energía como desencadenante. En su muestra, el antecedente traumático no se asoció significativamente con peores resultados. Los factores que tuvieron una asociación significativa fueron: el estado neurológico preoperatorio, la magnitud de la compresión medular, diabetes mellitus y el aumento de la señal medular en la $\mathrm{RM}$ inicial. ${ }^{28} \mathrm{Re}-$ cientemente Hollenberg y cols. llevaron a cabo un estudio similar en una cohorte de pacientes norteamericanos y comunicaron, como hallazgo significativo, peores valores neurológicos motores iniciales y posoperatorios en pacientes con lesión medular aguda comparados con aquellos con mielopatía. ${ }^{27}$

\section{DISCUSIÓN}

Presentamos una serie de cinco casos de trauma medular cervical asociado a OLLP que, si bien representa una cantidad baja de pacientes comparada con las de publicaciones previas, tiene como único precedente un reporte de caso de esta asociación por parte de autores de habla hispana. ${ }^{22}$ Como antecedente adicional destacable, Cabrera Rendón y cols. publicaron un caso de mielopatía asociada a OLLP. ${ }^{31}$

En nuestro medio, se desconoce la prevalencia de la OLLP en la lesión medular aguda traumática sin evidencia de fractura. Según publicaciones asiáticas, el 34\% de estas lesiones se presentan asociadas a OLLP. ${ }^{10}$ Bazán y cols. comunicaron una serie de 13 pacientes con lesión medular aguda traumática sin signos radiográficos y tomográficos de trauma, ninguno de ellos tenía OLLP. ${ }^{32}$

El perfil clínico de este cuadro incluye a pacientes de edad avanzada con antecedentes traumáticos menores. ${ }^{11}$ Dos de nuestros casos habían sufrido caídas de su propia altura como episodio traumático.

De acuerdo con la bibliografía consultada, los pacientes con OLLP, además del posible desarrollo de una mielopatía crónica, tienen un mayor riesgo de sufrir una lesión medular traumática. ${ }^{29} \mathrm{El}$ antecedente traumático no tendría impacto directo en los resultados comparado con otros factores que, se sabe, tienen una asociación significativa, como la gravedad del estado neurológico inicial, la edad avanzada, el aumento de la intensidad de la señal medular en la RM y la magnitud de la compresión. ${ }^{19,26-29}$ El tratamiento sugerido es la liberación quirúrgica precoz, y predominan los reportes del abordaje posterior (laminectomía o laminoplastia). ${ }^{11,18-20,25-30}$

En nuestra serie, el tratamiento quirúrgico de urgencia no proporcionó beneficios en función de la recuperación neurológica y la tasa de mortalidad en el posoperatorio inmediato fue alta. Cabe destacar que todos los casos incluidos tenían un puntaje ASIA elevado al ingresar (4 ASIA A y 1 C). Clínicamente cuatro pacientes tenían lesión medular completa y uno, síndrome medular central. Asimismo, en concordancia con los factores asociados con peores resultados en la bibliografía, todos nuestros casos se asociaron con imágenes medulares hiperintensas extensas en la RM preoperatoria y evidencia de estenosis del canal vertebral significativa.

Las principales debilidades de nuestro estudio son su carácter descriptivo y el bajo número de casos, por lo cual el aporte de los datos de la serie es limitado. No obstante, lo consideramos un reporte novedoso en la bibliografía de origen hispanoamericano sobre una asociación poco frecuente en nuestra región.

\section{CONCLUSIÓN}

Presentamos una serie de pacientes con trauma medular cervical asociado a OLLP infrecuente en nuestro medio, solo hay un reporte de caso registrado en la bibliografía hispanoamericana. La bibliografía reciente contempla la liberación quirúrgica por vía posterior a tiempo como tratamiento predominante. La lesión neurológica inicial severa, la edad avanzada, el aumento de la intensidad de la señal medular en la RM y la elevada compresión medular se relacionan con pobres resultados posoperatorios.

Conflicto de intereses: Los autores no declaran conflictos de intereses.

ORCID de I. Garfinkel: https://orcid.org/0000-0001-9557-0740

ORCID de G. Carrioli: https://orcid.org/0000-0003-4160-9712

ORCID de D. O. Ricciardi: https://orcid.org/0000-0002-1396-9115 


\section{BIBLIOGRAFÍA}

1. Ahuja CS, Schroeder GD, Vaccaro AR, Fehlings MG. Spinal cord injury -What are the controversies? J Orthop Trauma 2017;31(Suppl 4):S7-S13. https://doi.org/10.1097/BOT.0000000000000943

2. Krueger H, Noonan VK, Trenaman LM, Joshi P, Rivers CS. The economic burden of traumatic spinal cord injury in Canada. Chronic Dis Inj Can 2013;33(3):113-22. PMID: 23735450

3. Walters BC, Hadley MN, Hurlbert RJ, Aarabi B, Dhall SS, Gelb DE, et al; American Association of Neurological Surgeons; Congress of Neurological Surgeons. Guidelines for the management of acute cervical spine and spinal cord injuries: 2013 update. Neurosurgery 2013;60(CN_suppl_1):82-91. https://doi.org/10.1227/01.neu.0000430319.32247.7f

4. Boese CK, Lechler P. Spinal cord injury without radiologic abnormalities in adults: a systematic review. J Trauma Acute Care Surg 2013;75(2):320-30. https://doi.org/10.1097/TA.0b013e31829243c9

5. Pang D, Wilberger JE Jr. Spinal cord injury without radiographic abnormalities in children. J Neurosurg 1982;57(1):114-29. https://doi.org/10.3171/jns.1982.57.1.0114

6. Gupta SK, Rajeev K, Khosla VK, Sharma BS, Paramjit, Mathuriya SN, et al. Spinal cord injury without radiographic abnormality in adults. Spinal Cord 1999;37(10):726-9. https://doi.org/10.1038/sj.sc.3100900

7. Hendey GW, Wolfson AB, Mower WR, Hoffman JR; National Emergency X-Radiography Utilization Study Group. Spinal cord injury without radiographic abnormality: results of the National Emergency X-Radiography Utilization Study in blunt cervical trauma. J Trauma 2002;53(1):1-4. Disponible en: https://www.researchgate.net/profile/ Jerome_Hoffman2/publication/49600544_SCIWORA_is_Not_Just_Child's_Play_Analysis_of_the_NEXUS_Data/ links/54171d200cf2218008bed0fd/SCIWORA-is-Not-Just-Childs-Play-Analysis-of-the-NEXUS-Data.pdf

8. Como JJ, Samia H, Nemunaitis GA, Jain V, Anderson JS, Malangoni MA, et al. The misapplication of the term spinal cord injury without radiographic abnormality (SCIWORA) in adults. J Trauma Acute Care Surg 2012;73(5):1261-6. https://doi.org/10.1097/TA.0b013e318265cd8c

9. Yucesoy K, Yuksel KZ. SCIWORA in MRI era. Clin Neurol Neurosurg 2008;110(5):429-33. https://doi.org/10.1016/j.clineuro.2008.02.004

10. Boody BS, Lendner M, Vaccaro AR. Ossification of the posterior longitudinal ligament in the cervical spine: a review. Int Orthop 2019;43(4):797-805. https://doi.org/10.1007/s00264-018-4106-5

11. Chikuda H, Seichi A, Takeshita K, Matsunaga S, Watanabe M, Nakagawa Y, et al. Acute cervical spinal cord injury complicated by preexisting ossification of the posterior longitudinal ligament: a multicenter study. Spine (Phila Pa 1976) 2011;36(18):1453-8. https://doi.org/10.1097/BRS.0b013e3181f49718

12. Endo S, Shimamura T, Nakae H, Takakuwa T, Yamada Y, Kasai T, et al. Cervical spinal cord injury associated with ossification of the posterior longitudinal ligament. Arch Orthop Trauma Surg 1994;113(4):218-21. https://doi.org/10.1007/BF00441836

13. Fujimura Y, Nakamura M, Toyama Y. Influence of minor trauma on surgical results in patients with cervical OPLL. J Spinal Cord 1998;11(1):16-20. PMID: 9493765

14. Hayashi K, Yone K, Ito H, Yanase M, Sakou T. MRI findings in patients with a cervical spinal cord injury who do not show radiographic evidence of a fracture or dislocation. Paraplegia 1995;33(4):212-5. https://doi:org/10.1038/sc.1995.47

15. Koyanagi I, Iwasaki Y, Hida K, Akino M, Imamura H, Abe H. Acute cervical cord injury without fracture or dislocation of the spinal column. J Neurosurg 2000;93(1 Suppl):15-20. https://doi.org/10.3171/spi.2000.93.1.0015

16. Mizuno J, Nakagawa H, Hashizume Y. Pathology of the spinal cord damaged by ossification of the posterior longitudinal ligament associated with spinal cord injury. Spinal Cord 1999;37(3):224-47. https://doi.org/10.1038/sj.sc.3100758

17. Koyanagi I, Iwasaki Y, Hida K, Imamura H, Fujimoto S, Akino M. Acute cervical cord injury associated with ossification of the posterior longitudinal ligament. Neurosurgery 2003;53(4):887-92. https://doi.org/10.1227/01.neu.0000083590.84053.cc

18. Gu Y, Chen L, Dong RB, Feng Y, Yang HL, Tang TS. Laminoplasty versus conservative treatment for acute cervical spinal cord injury caused by ossification of the posterior longitudinal ligament after minor trauma. Spine $J$ 2014;14(2):344-52. https://doi.org/10.1016/j.spinee.2013.06.083

19. Gu J, Guan F, Zhu L, Guan G, Chi Z, Li W, et al. Predictors of surgical outcome in acute spinal cord injury patients with cervical ossification of the posterior longitudinal ligament. World Neurosurg 2016;90:364-71. https://doi.org/10.1016/j.wneu.2016.03.005 
20. Onishi E, Sakamoto A, Murata S, Matsushita M. Risk factors for acute cervical spinal cord injury associated with ossification of the posterior longitudinal ligament. Spine (Phila Pa 1976) 2012;37(8):660-6. https://doi.org/10.1097/BRS.0b013e31822da1d5

21. Okada S, Maeda T, Ohkawa Y, Harimaya K, Saiwai H, Kumamaru H, et al. Does ossification of the posterior longitudinal ligament affect the neurological outcome after traumatic cervical cord injury? Spine (Phila Pa 1976) 2009;34(11):1148-52. https://doi.org/10.1097/BRS.0b013e31819e3215

22. Malpica RLM, Malpica RM, Fernández LCLG, Reyes LJ, Jiménez RHO. Osificación del ligamento longitudinal posterior. Rev Esp Med Quir 2012;17(2):150-4. Disponible en: https://www.medigraphic.com/pdfs/quirurgicas/rmq-2012/rmq122p.pdf

23. Kirshblum SC, Burns SP, Biering-Sorensen F, Donovan W, Graves DE, Jha A, et al. International standards for neurological classification of spinal cord injury (Revised 2011). J Spinal Cord Med 2013;34(6):535-46. https://doi.org/10.1179/204577211X13207446293695

24. Tsuyama N. Ossification of the posterior longitudinal ligament of the spine. Clin Orthop Relat Res 1984;(184):7184. PMID: 6423334

25. Yang D-1, Zhang Y-z, Shen Y, Cao J-m. Surgical management of cervical cord injury with ossification of the cervical posterior longitudinal ligament. Orthop Surg 2011;3(2):95-101. https://doi.org/10.1111/j.1757-7861.2011.00131.x

26. Kwon SY, Shin JJ, Lee JH, Cho WH. Prognostic factors for surgical outcome in spinal cord injury associated with ossification of the posterior longitudinal ligament (OPLL). J Orthop Surg Res 2015;10:94. https://doi.org/10.1186/s13018-015-0235-3

27. Hollenberg AM, Mesfin A. Ossification of the posterior longitudinal ligament in North American patients: Does presentation with spinal cord injury matter? World Neurosurg 2020;143:e581-e589. https://doi.org/10.1016/j.wneu.2020.08.020

28. Choi JH, Shin JJ, Kim TH, Shin HS, Hwang YS, Park SK. Does intramedullary signal intensity on MRI affect the surgical outcomes of patients with ossification of posterior longitudinal ligament? J Korean Neurosurg Soc 2014;56(2):121-9. https://doi.org/10.3340/jkns.2014.56.2.121

29. Wu JC, Chen YC, Liu L, Huang WC, Chen TJ, Lo SS, et al. Conservatively treated ossification of the posterior longitudinal ligament increases the risk of spinal cord injury: a nationwide cohort study. $J$ Neurotrauma 2012;29(3):462-8. https://doi.org/10.1089/neu.2011.2095

30. Li H, Jiang C. [Multivariate analysis of the operative effect on cervical spinal cord injury without fracture or dislocation]. Zhongguo Gu Shang 2020;33(2):158-65. [En chino] https://doi.org/10.12200/j.issn.1003-0034.2020.02.014

31. Cabrera Rendón N, Fernández Albán M, Figueredo Méndez J, Salazar López A. Osificación del ligamento longitudinal posterior cervical. Presentación de un caso y revisión de la literatura. CInvest Medicoquir 2020;12(2). Disponible en: http://revcimeq.sld.cu/index.php/imq/article/view/612

32. Bazán PL, Borri AE, Medina M. Predictors in adult SCIWORA. Coluna/Columna 2013;12(4):326-9. https://doi.org/10.1590/S1808-18512013000400014 